\title{
Determination of the Idle Mode of the Stage of Axial Turbine during Operation at Partial Loads
}

\author{
Aleksandr Shubenko1, Vladimir Goloshchapov¹, Daria Senetska ${ }^{1 *}$, Oleksandr Senetskyi \\ 1 Department of process optimization and turbomachinery designs, A. Podgorny Institute of Mechanical Engineering Problems of \\ the National Academy of Sciences of Ukraine, Pozhars'koho St. 2/10, 61046 Kharkiv, Ukraine \\ 2 Department of Renewable Energy, O.M. Beketov National University of Urban Economy in Kharkiv, Marshal Bazhanov Street 17 , \\ 61002 Kharkiv, Ukraine \\ * Corresponding author, e-mail: dashasenetskaya@ipmach.kharkov.ua
}

Received: 15 October 2020, Accepted: 19 October 2020, Published online: 02 December 2020

\begin{abstract}
On the basis of the one-dimensional theory of calculating the operation of steam turbine stages the method for determining the idle mode, the initial data for which are the geometric characteristics of the blade rows is proposed. Formulas have been obtained that make it possible to calculate the efficiency in the proximity of Rotor Blades (RB) at operating modes from nominal to the idle mode, depending on the flow rate of the stage and taking into account the use of kinetic energy with the runaway velocity and losses arising at off-design angles of flow on the Rotor Blade (RB). The results of possible computational studies and their comparison for an incompressible working fluid with the results of experimental studies showed the good possibilities of the proposed method for determining the idle mode in a wide range of the characteristics of stages.
\end{abstract}

Keywords

turbine stage, geometric and energy characteristics of rows, efficiency at the row stage, the idle mode, the relative flow rate

\section{Introduction}

Currently, one of the most acute problems in the Ukrainian electric power industry is the shortage of maneuverable capacity, which is provided by the power units of thermal power plants. Regulation of daily schedules of electricity consumption has complicated the operating modes of thermal power plants and has led to an increase in the number of powerful units which are put into reserve, deepening their nights unloading and an increased number of shutdowns on weekends. All this causes accelerated wear of equipment and excessive fuel consumption when operating in low-efficiency modes at partial loads. This applies to both steam and hydraulic turbines [1-3]. Special attention should be paid to the problems of efficiency and reliability of the power units of combined heat and power plants, operated according to the thermal schedule the prevailing time, in which the stages of Low-Pressure Cylinder (LPC) operate in the area of low-flow rate modes [4].

Note that under low-flow rate modes, according to the definition used in $[5,6]$, mean modes below the idle, accompanied by the emergence of complex physical phenomena in the stage, namely, the formation of various vortex structures that significantly affect both the increase in energy losses and vibration state of the Rotor Blades. Thus, in order to determine the efficient and safe operation of turbine plants at thermal power plants and combined heat and power plants (taking into account more detailed studies of gas-dynamic and thermal processes in a turbine), an important role is played by determining the idle mode of turbine stage when it is operating at partial loads.

\section{Determination of the idle mode}

In the monograph of the authors [6], some empirical approaches to the determination of the idle mode of turbine stage, based on the processing of available experimental data, are considered. Their advantages include simplicity and their disadvantages - limited application only in the range of the geometric characteristics of stages used in the experiment.

Therefore, another approach to the determination of the idle mode which based on a simplified analysis of thermal processes occurring in an axial turbine stage is interested. This will make it possible to determine not only the actual idle mode, but also the change in the stage 
parameters (such as the efficiency $\eta_{u}$, the degree of reactivity $\rho$, the characteristic number $u / C_{0}$ etc.) during operating modes from nominal to idle.

To solve this problem, let us consider some basic definitions necessary for calculating thermal processes in an axial turbine stage (Fig. 1) [7].

In the Stator Blades (SB) of the stage, there is an acceleration of the flow of the working fluid entering the stage with the velocity $c_{0}$ in the section $0-0$ (Fig. 1 (a)), with a decrease in the pressure. In this case, the velocity $c_{1}$ and the pressure $P_{1}$, the values of which depend on the ratio of the throat areas at the outlet channels of SB and the Rotor Blades (RB) are set in a rim clearance (section 1-1).

In the channels of RB there is a turn of the flow leaving $\mathrm{SB}$ and its acceleration when the pressure decreases from $P_{1}$ to $P_{2}$.

The power of the axial stage on the row of RB, according to the Euler equation, is determined both by the flow rate of working fluid through the stage and by the rotation of the flow in RB and can be expressed by the dependence $N_{u}=G\left(u_{1} \times c_{1 u}+u_{2} \times c_{2 u}\right)$, where $G$ is the flow rate of the working fluid; $u$ is the peripheral velocity in the inlet and outlet sections of RB of the axial stage at $d_{1}$ and $d_{2}$ for sections 1 and $2 ; c_{1 u}, c_{2 u}$ are the projections of absolute velocity of the working fluid exit from SB (index 1) and $\mathrm{RB}$ (index 2) on the circumferential direction.

Based on the movement of the working fluid on coaxial cylindrical (or close to them) surfaces and assuming that for the axial stage $u_{1} \approx u_{2} \approx u$, the specific energy (work) for the stage can be represented as

$\frac{N_{u}}{G}=H_{u}=u\left(c_{1 u}+c_{2 u}\right)=u\left(w_{1 u}+w_{2 u}\right)$,
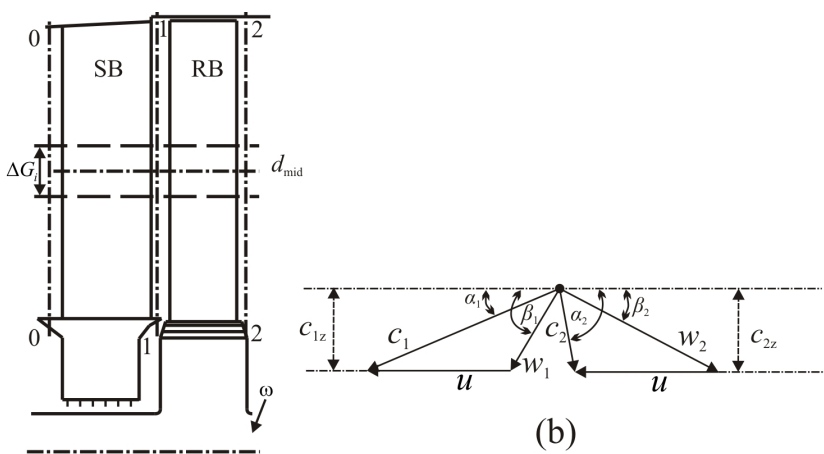

(a)

(b)

Fig. 1 Schematic representation of an axial turbine stage (a) and velocity triangles (b) where $w_{1 u}$ and $w_{2 u}$ are the relative flow rates at the inlet (index 1) and outlet (index 2) of RB (Fig. 1 (b)).

The expression for the specific energy Eq. (1) can be written in another way - when considering the thermal process (Fig. 2) in the turbine stage.

Indeed, theoretically, in the stage from one kilogram of the working fluid, it is possible to obtain work with an isentropic flow which equals to the available heat drop, determined by the parameters of complete stagnation $\bar{H}_{0}=\bar{h}_{0}-h_{2 t}$.

The relationship between the parameters of the working fluid and the enthalpy $h$ is determined by the dependence obtained for an ideal gas

$$
\begin{aligned}
& \bar{h}_{0}-h_{2 t}=\bar{H}_{0}=\frac{\kappa}{\kappa-1} \bar{P}_{0} \bar{v}_{0}\left(1-\left(\frac{P_{2}}{\bar{P}_{0}}\right)^{\frac{\kappa-1}{\kappa}}\right), \\
& =\frac{\kappa}{\kappa-1}\left(\bar{P}_{0} \bar{v}_{0}-P_{2} v_{2 t}\right),
\end{aligned}
$$

where $\kappa$ is the isentropic exponent; for air $\kappa=1.4$; for the superheated steam $\kappa=1.3$; for the dry-saturated steam $\kappa=1.135$.

The movement of the working fluid in the blades, taking into account the flow velocities in $\mathrm{RB} w_{1}$ at the inlet and $w_{2}$ at the outlet, and the peripheral velocity $u$, can be represented in the form of velocity triangles (Fig. 1 (b)): $\vec{c}_{1}=\vec{u}+\vec{w}_{1}$ for the trailing edges of the Stator Blades (section 1-1) and $\vec{c}_{2}=\vec{u}+\vec{w}_{2}$ (section 2-2) for the trailing edges of the Rotor Blades. Velocities triangles are considered for an elementary stream, the position of which in the control sections $(0-0,1-1,2-2)$ depends on the values of the exit angles $\alpha_{1}$ for SB and $\beta_{2}$ for RB.

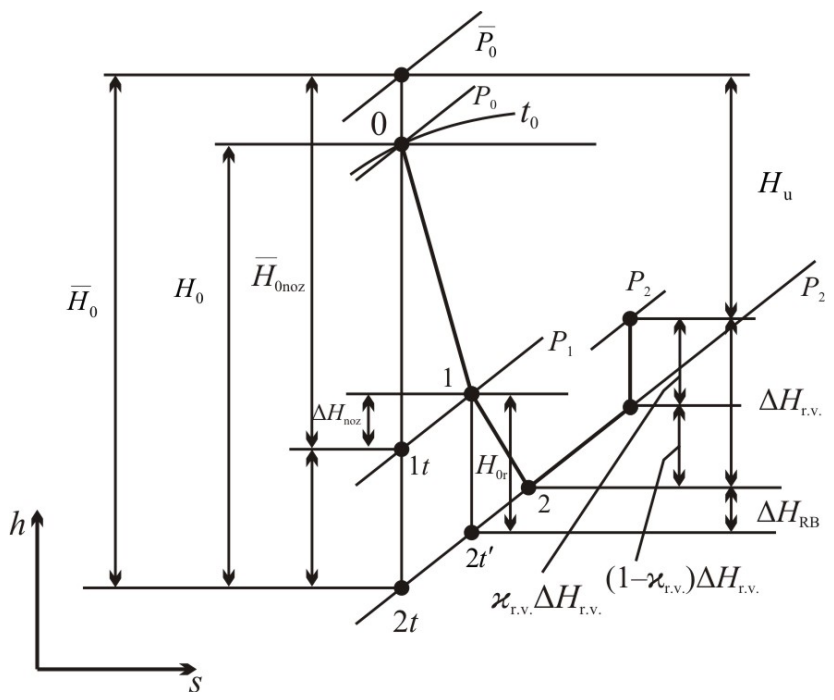

Fig. 2 Thermal process of the turbine stage 
A number of relationships follow from the velocities triangles:

$$
\begin{aligned}
& c_{1 u}=c_{1} \times \cos \alpha_{1}, \quad w_{1 u}=w_{1} \times \cos \beta_{1} ; \\
& c_{1 z}=c_{1} \times \sin \alpha_{1}, \quad w_{1 z}=w_{1} \times \sin \beta_{1}=c_{1 z} ; \\
& c_{2 u}=c_{2} \times \cos \alpha_{2}, \quad w_{2 u}=w_{2} \times \cos \beta_{2} ; \\
& c_{2 z}=c_{2} \times \sin \alpha_{2}, \quad w_{2 z}=w_{2} \times \sin \beta_{2}=c_{2 z} ; \\
& w_{1}^{2}=c_{1}^{2}+u^{2}-2 u c_{1} \times \cos \alpha_{1} ; \\
& c_{2}^{2}=w_{2}^{2}+u^{2}-2 u w_{2} \times \cos \beta_{2} .
\end{aligned}
$$

As a characteristic of the operating mode of axial stage, the fictitious velocity $C_{0}$ is taken, determined by the value of the heat drop $\bar{H}_{0}$ as

$$
\bar{H}_{0}=\frac{C_{0}^{2}}{2} \text { or } C_{0}=\sqrt{2 \bar{H}_{0}} \text {. }
$$

The isentropic heat drop $\bar{H}_{0}$ is a theoretical value corresponding to the absence of energy losses in SB nozzles $\xi_{\mathrm{SB}}=0$ and in RB channels $\xi_{\mathrm{RB}}=0$.

When a stage operates on a real working fluid, the friction losses and the vortex formation (end losses) are formed, which determining for an elementary (onedimensional) flow through the velocity coefficients: for SB nozzles $\varphi=c_{1} / c_{1 t}$, for RB channels $\psi=w_{2} / w_{2 t}$, where $c_{1 t}=\sqrt{2 \bar{H}_{0 \text { noz }}}, w_{2 t}=\sqrt{w_{1}^{2}+H_{0 r}}, \bar{H}_{0 \text { noz }}$ and $H_{0 r}$ is determined according to $h$-S diagram (Fig. 2).

To distribute the heat drop of stage $\bar{H}_{0}$ between SB and $\mathrm{RB}$, the concept of the stage reactivity degree $\rho$ is introduced as the ratio of the isentropic heat drop triggered in RB to the isentropic heat drop of stage $\rho=\frac{H_{0 r}}{\bar{H}_{0}}$.

Taking into account the reactivity degree and losses, the flow rate of working fluid from the nozzles $c_{1}$ is determined as $c_{1}=\varphi \sqrt{2 \bar{H}_{0}(1-\rho)}$

and the energy loss in the nozzles

$$
\Delta H_{n o z}=\frac{c_{1 t}^{2}-c_{1}^{2}}{2}=\frac{c_{1 t}^{2}}{2}\left(1-\varphi^{2}\right)=\xi_{S B} \times \frac{c_{1 t}^{2}}{2} .
$$

The rate of outflow from $\mathrm{RB}$ channels, taking into account the rate coefficient, is expressed by

$$
w_{2}=\psi \sqrt{2 \rho \bar{H}_{0}+w_{1}^{2}}
$$

and the energy loss in RB

$$
\Delta H_{\mathrm{RB}}=\frac{1}{2}\left(w_{2 t}^{2}-w_{2}^{2}\right)=\frac{w_{2 t}^{2}}{2}\left(1-\psi^{2}\right)
$$

Then $H_{u}=\bar{H}_{0}-\Delta H_{n o z}-\Delta H_{\mathrm{RB}}-\Delta H_{\text {r.v. }}$, where $\bar{H}_{0}$, $\Delta H_{n o z}, \Delta H_{\mathrm{RB}}$ are defined by Eqs. (2), (6), (8); $\Delta H_{\text {r.v. }}$ is the energy loss with output velocity, equals to $\Delta H_{\mathrm{r} . \mathrm{v} .}=\frac{C_{2}^{2}}{2}$.

The main characteristic that determines the efficiency operate of stage is the coefficient of efficiency $\eta_{u}$ is the ratio of the power generated by the flow on the row of RB $N_{u}$ to the available power of stage $\bar{N}_{0}$.

Considering that $\bar{N}_{0}=G \times\left(\bar{H}_{0}-\varkappa_{\text {r.v. }} \Delta H_{\text {r.v. }}\right) ; N_{u}=G \times H_{u}$, where $\varkappa_{\text {r.v. }}$ is the coefficient of kinetic energy utilization with runaway velocity in the next stage [7], we have for $\eta_{u}$

$\eta_{u}=\frac{H_{u}}{\bar{H}_{0}-\varkappa_{\text {r.v. }} \Delta H_{\text {r.v. }}}=f\left(\alpha_{1}, \beta_{2}, \rho, \varphi, \psi, u / C_{0}\right)$.

Let us write further Eq. (9) in a form convenient for analyzing the variable operating modes of stage and determining the idle mode.

Keep in mind that the equality of the end areas of SB and $\mathrm{RB}$ is accepted then the axial stage can be considered in the first approximation in a one-dimensional formulation, taking the velocity coefficients as integrals over the length of blades. In addition, we will restrict ourselves to considering the flow of an incompressible working fluid, which is quite well observed near the idle mode even for stages of large fanning $\Theta=l / d_{\text {mid }}[6]$.

Then the dependence $\eta_{u}$ allowing for Eqs. (1), (3), (4) will be written as follows

$$
\eta_{u}=\frac{2 u\left(c_{1} \cos \alpha_{1}+w_{2} \cos \beta_{2}-u\right)}{C_{0}^{2}\left(1-\varkappa_{\text {r.v. }} \frac{c_{2}^{2}}{C_{0}^{2}}\right)} .
$$

Finally, after simple transformations with substitution in Eq. (10) of the values $c_{1}, c_{2}$ and $w_{2}$ from Eqs. (3), (5) and (7) we obtain allowing for the assumptions

$$
\frac{w_{2}}{c_{1}} \approx \frac{\sin \alpha_{1}}{\sin \beta_{2}}
$$

$\eta_{u}=\frac{2 u / C_{0}\left(\varphi \cos \alpha_{1} \sqrt{1-\rho}-u / C_{0}+\varphi \operatorname{ctg} \beta_{2} \times \sin \alpha_{1} \sqrt{1-\rho}\right)}{1-\varkappa_{\text {r.v. }}\left(A^{2}+\left(u / C_{0}\right)^{2}-2\left(u / C_{0}\right) \times A \times \cos \beta_{2}\right)}$,

where $A=\varphi \frac{\sin \alpha_{1}}{\sin \beta_{2}} \sqrt{1-\rho}$.

The stage reactivity degree can be determined from Eq. (11), which transformed by substituting $c_{1}$ and $w_{2}$ to the form 


$$
\begin{aligned}
& \frac{\psi^{2}}{\varphi^{2}(1-\rho)} \\
& \times \sqrt{\left(\varphi^{2}(1-\rho)+\rho+\left(u / C_{0}\right)^{2}-2 \varphi\left(u / C_{0}\right) \sqrt{1-\rho} \times \cos \alpha_{1}\right)} \\
& =\frac{\sin ^{2} \alpha_{1}}{\sin ^{2} \beta_{2}} .
\end{aligned}
$$

The operation mode of stage when using Eqs. (12) and (13) is set by a value $u / C_{0}$ that is interconnected with the relative volumetric flow [8] which determined by the expression $\overline{G v_{2}}=\frac{\overline{G v_{2}}}{\left(\overline{G v_{2}}\right)_{\text {nот }}}$, where $v$ is the specific volume of working fluid; $\overline{G v_{2}}$ and $\left(\overline{G v_{2}}\right)_{\text {nom }}$ are the volumetric flow rate at investigated and nominal operating modes, respectively.

A more convenient form of determining the mode is to use the flow coefficient $\bar{c}_{z}=c_{z} / u$ instead of $u / C_{0}$ [9].

ThenEqs.(12)and(13)canbetransformed to the following form, taking into account that $\frac{u}{C_{0}}=\frac{\sin \alpha_{1} \times \varphi \sqrt{1-\rho}}{\bar{c}_{z}}$,

$$
\eta_{u}=\frac{2 \frac{\sin ^{2} \alpha_{1} \times \varphi^{2}(1-\rho)}{\bar{c}_{z}}\left(\operatorname{ctg} \alpha_{1}-\left(1 / \bar{c}_{z}\right)+\operatorname{ctg} \beta_{2}\right)}{1-\varkappa_{\mathrm{r} . \mathrm{f}} A^{2}\left(1+\frac{\sin ^{2} \beta_{2}}{\bar{c}_{z}^{2}}-2 \frac{\sin \beta_{2}}{\bar{c}_{z}} \times \cos \beta_{2}\right)} .
$$

The value of the stage reactivity degree can be represented as

$$
\rho=\frac{\varphi^{2} B}{1+\varphi^{2} B},
$$

where $B=\frac{1}{\psi^{2}} \times \frac{\sin ^{2} \alpha_{1}}{\sin ^{2} \beta_{2}}-\frac{\sin ^{2} \alpha_{1}}{\bar{c}_{z}^{2}}+2 \frac{\sin \alpha_{1} \cos \alpha_{1}}{\bar{c}_{z}}-1$.

It is obvious that $\bar{c}_{z}$, corresponding to the idle mode of stage, when $\eta_{u}=0$ is determined by the values of angles $\alpha_{1}$ and $\beta_{2}$. Note that under the assumptions made the relationship between $\bar{c}_{z}$ and $\overline{G v_{2}}$ is established by a simple expression $\bar{c}_{z} /\left(\bar{c}_{z}\right)_{\text {nom }}=\overline{G v_{2}}$.

\section{Estimates of the variable operating modes of turbine stages}

Let us further make some estimates of the variable operating modes of stages with different geometric characteristics. As an example, let us consider the stages of K-800-240 turbine, the thermal calculation of which is given at [7]: the stage No. 4 is the first section of the High Pressure
Cylinder (HPC); the stage No. 14 is the section of the Intermediate Pressure Cylinder (IPC) and the stage No. 22 is the section of LPC. The velocity coefficients $\varphi$ and $\psi$ for the nominal mode are selected from the thermal calculation of turbine, namely $\varphi=0.97, \psi=0.95$ (the stage No. 4); $\varphi=0.97, \psi=0.955$ (the stage No. 14); $\varphi=0.973, \psi=0.956$ (the stage No. 22). Changes in the angles $\alpha_{1}$ and $\beta_{2}$ over the stages in the turbine cylinders are shown in Fig. 3.

As for the utilization coefficient of runaway velocity $\varkappa_{\text {r.v. }}$, in most cases, when the heat calculation of stage $\varkappa_{\text {r.v. }}$ is assumed to be either 0 (when considering the last stages of the compartments, when the runaway velocity is completely lost), or 1 - for intermediate stages.

Note that the energy losses that form the velocity coefficients $\varphi$ and $\psi$ can be attributed to the losses associated with the flow around the blades, namely, profile, end, losses from unsteadiness. For variable operating modes, a significant role is played by the losses arising at off-design angles of flow on the cascade, the so-called shock losses.

In this case, in the first approximation, we restrict ourselves to taking into account the shock losses in RB, assuming that they appear much weaker in SB.

Without analyzing here in detail the issue of changes in energy losses in a stage under off-design operating modes, which is the subject of independent research and is largely considered in the literature (see, for example, [10]), we restrict ourselves to approximate estimates of changes in losses by generalizing the results of experimental studies by various authors, executed for example by Professor I. V. Kotlyar [11].

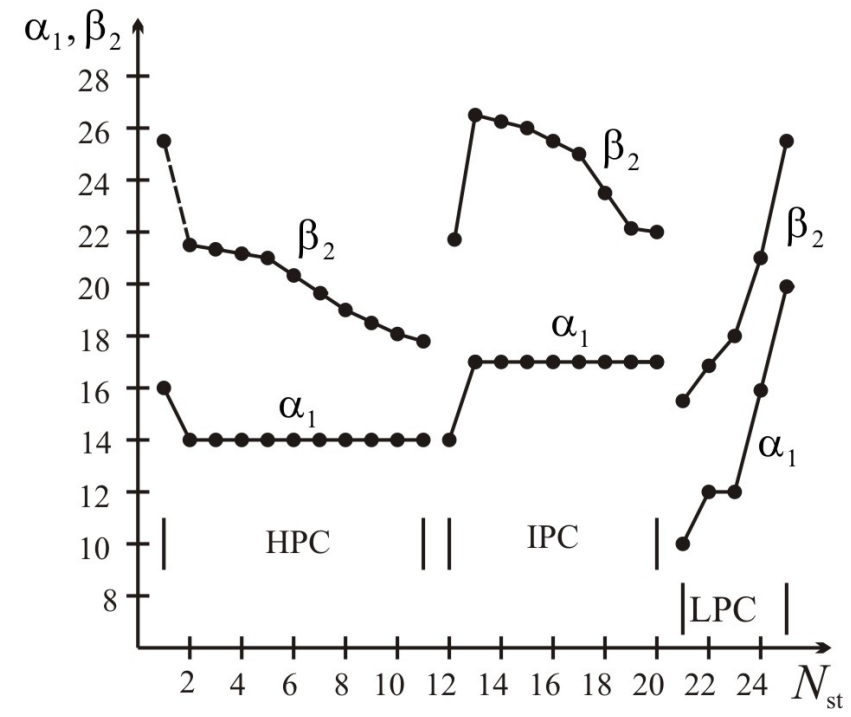

Fig. 3 Angles of flow exit in the stages of turbine K-800-240 (Leningrad Machine-Building Plant) 
The resulting dependence can be written as follows (Fig. 4) $\frac{\psi}{\psi_{\text {nom }}}=1.01-0.01\left(\frac{\beta_{1}}{\beta_{1 \text { nom }}}\right)^{3}$, where $\beta_{1}$ is determined from the expression

$$
\sin \beta_{1}=\frac{\bar{c}_{z} u}{w_{1}}=\frac{\bar{c}_{z}}{\sqrt{\left(\frac{\bar{c}_{z}}{\sin \alpha_{1}}\right)^{2}+1-2 \bar{c}_{z} \operatorname{ctg} \alpha_{1}}} .
$$

Note that, in accordance with Eq. (16), when determining $\sin \beta_{1}$, the angle $\beta_{1}$ can take on a value either less or more than $90^{\circ}$. It is easy to make sure that at $\bar{c}_{z} \operatorname{ctg} \alpha_{1}>1$, $\beta_{1}<90^{\circ}$ and, conversely, at $\bar{c}_{z} \operatorname{ctg} \alpha_{1}<1, \beta_{1}>90^{\circ}$.

\section{Calculation research results}

Let us consider further some results of the computational study. In Figs. 5, 6 show the change in efficiency for three stages when changing the mode from the nominal to the idle mode for the cases $\varkappa=0$ and $\varkappa=1$ with and without taking into account the shock losses.

As you can see, at $\varkappa=0$ (Fig. 5), there is a smooth decrease in the efficiency from the maximum value at the nominal mode to $\eta_{u}=0$ at the idle mode. In this case, the nature of curves essentially depends on whether it is assumed that $\varphi, \psi=$ const or the shock losses are taken into account.

On the other hand, at $\varkappa=1$ (Fig. 6) and $\varphi, \psi=$ const $\eta_{u}$ changes little with decreasing $\bar{c}_{z}$ from the nominal value to modes close to the idle, and only near the idle drops sharply to zero. Taking into account the shock losses, the nature of change in the efficiency is close to the case $\varkappa=0$.

It is interesting to compare the values of $\bar{c}_{z i m}$ of the idle, calculated using the analytical dependence Eq. (14), with the results of calculations using empirical formulas obtained by the authors earlier [6].

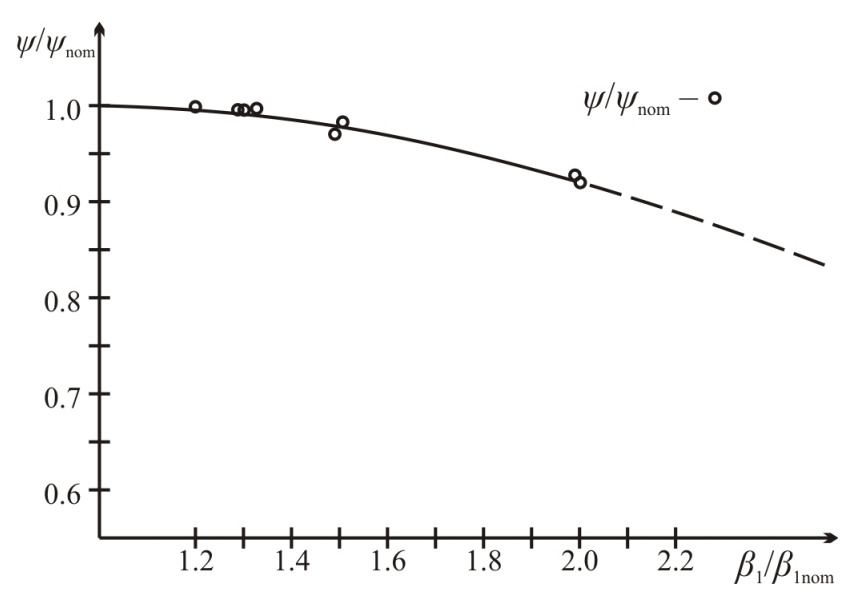

Fig. 4 Generalization of the results of experimental studies of changes in the velocity coefficient of SB from the entry angle of blade
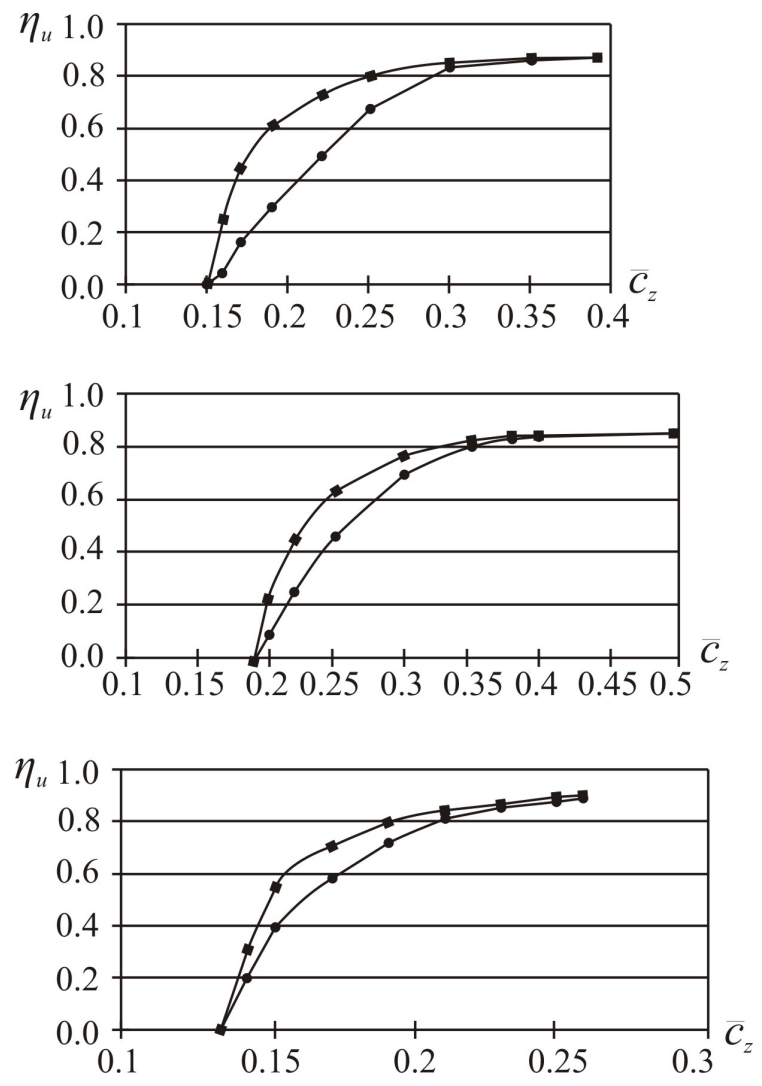

Fig. 5 The change in the efficiency of stages depending on the operating mode of turbine at $\varkappa=0$ : (a) - the stage No. 4;

(b) - the stage No. 14; (c) - the stage No. 22

Legend: black square $-\psi, \varphi=$ const;

black circle - taking into account the shock losses

Table 1 shows the values of $\bar{c}_{z \text { im }}$ for the three investigated stages, calculated by the analytical expression Eq. (14), as well as using the empirical formula (2.41) [6] and the formula (3.4) [6], obtained from the characteristics of nominal mode.

As can be seen from the table, the coincidence of the results according to the analytical formula Eq. (14) and the empirical formula (2.41) [6], which built according to the geometric characteristics of experimentally studied group of stages with the large fanning, cannot be considered satisfactory. This is due to the fact that formula (2.41) [6] was obtained on the basis of processing the results of experimental studies of a small number stages in a narrow range of variation of the bushing ratio values $\overline{\text { bush }}_{\text {bush }}=r_{\text {bus }} / R_{\text {out }}$, where $r_{\text {bush }}$ is the radius of bushing; $R_{\text {out }}$ is the outer radius of RB.

A significantly better the coincidence is observed when comparing the results according to analytical formula Eq. (14) and formula (3.4) [6], obtained from the characteristics of nominal mode, which allows them to be recommended for determining the values of $\bar{c}_{z i m}$ (or $\overline{G v}_{2}$ im ) in a wide range of the stage characteristics. 

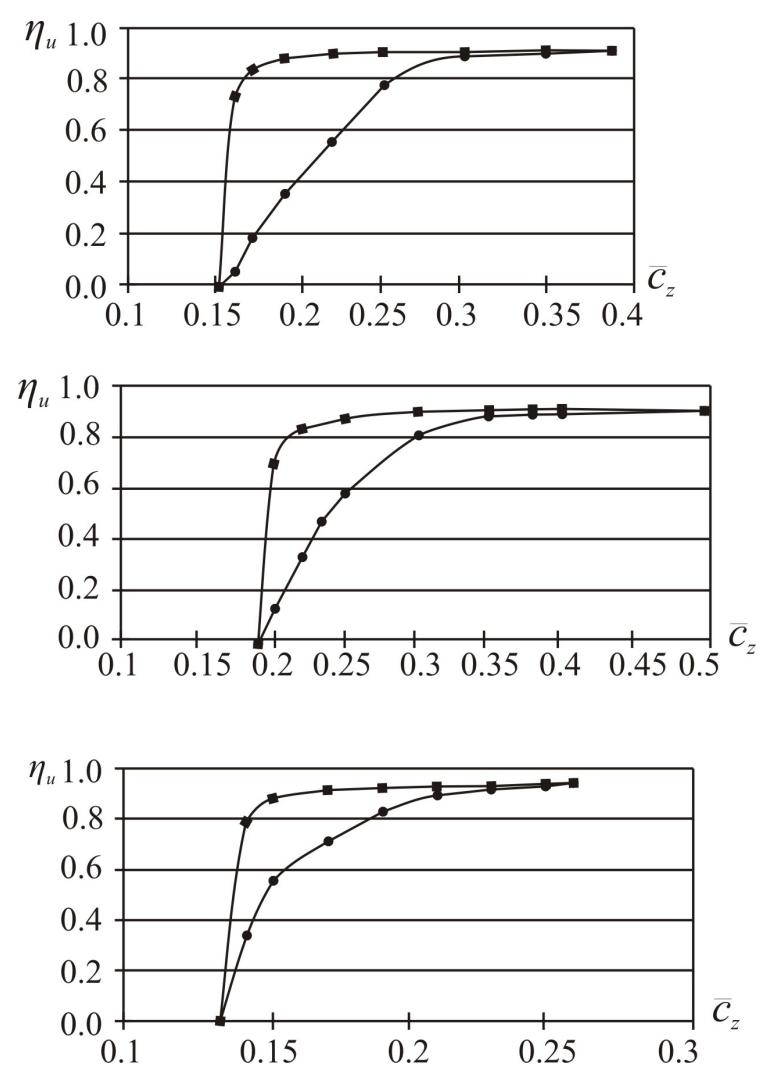

Fig. 6 The change in the efficiency of stages depending on the operating mode of turbine at $\varkappa=1:$ (a) - the stage No. 4;

(b) - the stage No. 14; (c) - the stage No. 22

Legend: black square $-\psi, \varphi=$ const;

black circle - taking into account the shock losses

Table 1 Values of $\bar{c}_{z \text { im }}$ for three stages, obtained by the different formulas

\begin{tabular}{lccc}
\hline No. stage & No. 4 & No. 14 & No. 22 \\
\hline $\begin{array}{l}\text { Obtained by the analytical } \\
\text { formula Eq. (14) }\end{array}$ & 0.151 & 0.188 & 0.125 \\
$\begin{array}{l}\text { Obtained by the geometric } \\
\text { characteristics, formula (2.41) [6] }\end{array}$ & 0.096 & 0.121 & 0.054 \\
$\begin{array}{l}\text { Obtained by the characteristics of } \\
\text { nominal mode, formula (3.4) [6] }\end{array}$ & 0.171 & 0.21 & 0.104 \\
\hline
\end{tabular}

\section{References}

[1] Karakurt, S. A., Güneş, Ü. "Performance Analysis of a Steam Turbine Power Plant at Part Load Conditions", Journal of Thermal Engineering, 3(2), pp. 1121-1128, 2017. https://doi.org/10.18186/thermal.298611

[2] Arakelyan, E. K, Pikina, G. A., Andryushin, A. V, Mezin, S. V., Andryushin, K. A., Kosoy, A. A., Pashchenko, F. F. "Features of steam turbine stages operation in low-flow modes when modeling hydrodynamic processes in the turbine in steamless and motor modes", Procedia Computer Science, 170, pp. 935-940, 2020. https://doi.org/10.1016/j.procs.2020.03.105

\section{Conclusion}

1. An analytical approach to obtaining the dependence for determining the circumferential efficiency $\eta_{u}$ at variable operating modes of turbine from the nominal to the idle mode is considered.

When working out the dependence, it was taken into account that the utilization coefficient of the runaway velocity $\varkappa$ can take on the value of either 0 or 1 , as well as the change in the velocity coefficient $\psi$ when considering that the shock losses.

2. A decrease in the operation modes of stage characterized by a flow rate $\bar{c}_{z}=c_{z} / u$ below the no-load value, leads to a qualitative change in the gas-dynamic processes in the stage, when analytical approaches become unacceptable and the results of targeted experimental studies are needed.

3. A comparison of the values of the flow rate coefficients characterizing the no-load mode of various stages and calculated using the analytical formula Eq. (14) with the results of calculation using empirical dependences obtained by the authors earlier, showed that the best coincidence is observed when comparing the calculation results using formulas Eq. (14) and (3.4) [6], obtained from the characteristics of nominal mode.

[3] Kim, S.-J., Suh, J.-W., Choi, Y.-S., Park, J., Park, N.-H., Kim, J.-H. "Inter-Blade Vortex and Vortex Rope Characteristics of a PumpTurbine in Turbine Mode under Low Flow Rate Conditions", Water, 11(12), Article number: 2554, 2019. https://doi.org/10.3390/w11122554

[4] Shubenko, A. L., Goloshchapov, V. N., Senetska, D. O. "The operation of the last stage of steam turbine at the low-flow rate modes", Energetika, 66(1), pp. 58-67, 2020. https://doi.org/10.6001/energetika.v66i1.4299 
[5] Khaimov, V. А. "Малорасходные режимы ЦНД турбины Т-250/300-240" (Little consumable modes of low-pressure of T-250/300-240 turbine), BHV-Petersburg, St. Petersburg, Russia, 2007. (in Russian)

[6] Shubenko, A. L., Goloshchapov, V. N., Bystritsky, L. N., Agafonov, B. N., Alekhina, S. V., Kasilov, V. I. "Паровые турбины: малорасходные режимы ступеней низкого давления" (Steam turbines: little consumable modes of low pressure stages), Energotech, St. Petersburg, Russia, 2018. (in Russian)

[7] Kostyuk, A. G., Frolov, V. V. "Турбины тепловых и атомных электростанций" (Turbines of thermal and nuclear power plants), MPEI, Moscow, Russia, 2001. (in Russian)

[8] Kapinos, V. M., Garkusha, A. V. "Переменный режим работы паровых турбин" (Variable mode of operation of steam turbines), Higher school, Kiev, Ukraine, 1989. (in Russian)
[9] Kirillov, I. I. "Теория турбомашин" (The theory of turbomachines), Mechanical engineering, St. Petersburg, Russia, 1972. (in Russian)

[10] Samoilovich, G. S., Troyanovsky, V. М. "Переменные и переходные режимы в паровых турбинах" (Variables and transient modes in steam turbines), Energoizdat, Moscow, Russia, 1982. (in Russian)

[11] Kotlyar, I. V. "Переменный режим работы газотурбинных установок" (Variable operating mode of gas turbine installations), Mashgiz, Moscow, Russia, 1961. (in Russian) 